\section{Oral diagnosis and treatment planning: part 1. Introduction}

\author{
P. Newsome, ${ }^{1}$ R. Smales ${ }^{2}$ and K. Yip ${ }^{3}$
}

\author{
VERIFIABLE CPD PAPER
}

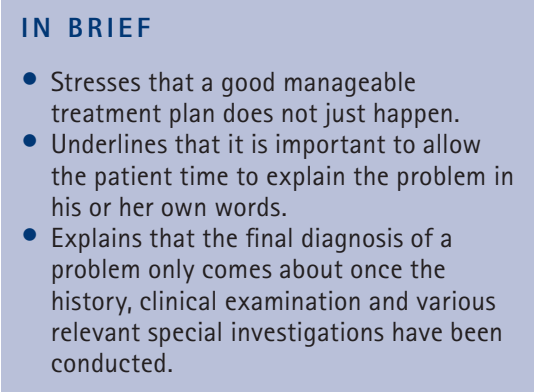

\begin{abstract}
A good manageable treatment plan does not just happen, but comes about as the natural consequence of taking carefully considered steps. History taking and clinical examination are two of the most important aspects of the patient assessment process, and complement each other to such an extent that it is impossible to build a satisfactory treatment plan without combining and collating information from the two procedures.
\end{abstract}

\section{INTRODUCTION}

A good manageable treatment plan does not just happen, but comes about as the natural consequence of taking the following carefully considered four steps:

- Collect and collate all of the relevant information obtained by means of the case history and examination to

\begin{tabular}{|c|c|}
\hline \multicolumn{2}{|c|}{$\begin{array}{l}\text { ORAL DIAGNOSIS } \\
\text { AND TREATMENT PLANNING* }\end{array}$} \\
\hline Part 1. & $\begin{array}{l}\text { Introduction to oral diagnosis } \\
\text { and treatment planning }\end{array}$ \\
\hline Part 2. & Dental caries and assessment of risk \\
\hline Part 3. & $\begin{array}{l}\text { Periodontal disease and } \\
\text { assessment of risk }\end{array}$ \\
\hline Part 4. & $\begin{array}{l}\text { Non-carious tooth surface loss } \\
\text { and assessment of risk }\end{array}$ \\
\hline Part 5. & $\begin{array}{l}\text { Preventive and treatment } \\
\text { planning for dental caries }\end{array}$ \\
\hline Part 6. & $\begin{array}{l}\text { Preventive and treatment } \\
\text { planning for periodontal disease }\end{array}$ \\
\hline Part 7. & $\begin{array}{l}\text { Treatment planning for } \\
\text { missing teeth }\end{array}$ \\
\hline Part 8. & $\begin{array}{l}\text { Reviews and maintenance } \\
\text { of restorations }\end{array}$ \\
\hline \multicolumn{2}{|c|}{$\begin{array}{l}\text { *This series represents chapters } 1,7,8,9,14,15,16 \text { and } 19 \text { from } \\
\text { the BDJ book AClinical Guide to Oral I Diagnosis and Treatment } \\
\text { Planning. All other chapters are published in the complete } \\
\text { clinical guide available from the } B D J \text { Books online shop. }\end{array}$} \\
\hline
\end{tabular}

Associate Professor, Comprehensive Dental Care, Faculty of Dentistry, The University of Hong Kong, Hong Kong: ${ }^{2 *}$ Visiting Research Fellow, School of Dentistry, Faculty of Health Sciences, The University of Adelaide, Adelaide, South Australia 5005, Australia; ${ }^{3}$ Adjunct Professor, School of Dentistry, Charles Sturt University, Orange, New South Wales 2800, Australia

${ }^{*}$ Correspondence to: Roger J. Smales

Email: roger.smales@adelaide.edu.au

Accepted 7 June 2012

DOI: 10.1038/sj.bdj.2012.559

${ }^{\circledR}$ British Dental Journal 2012; 213: 15-19 identify a list of problems

- Establish the dental diagnosis after determining the need for any special tests and consultations

- Consider the various treatment options available and their likely priorities

- Formulate the treatment plan in consultation with the patient, which includes any initial emergency and disease control phases and then the probable final or definitive phase.

\section{FOUR STEPS TO TREATMENT PLANNING}

It is important to differentiate between a treatment plan that focuses on treatment at the individual tooth level, and a plan that formulates a longterm strategy involving decisions about treating teeth in the context of the rest of the dentition, and about managing the rest of the dentition in the context of the masticatory system and the individual. To be successful, any treatment plan must incorporate short-term, medium-term and longterm views, and must take a holistic view of the patient as a person with specific needs and not just a 'mouth to be fixed' or a 'case to be treated' in a predetermined manner. Successful treatment planning requires adequate knowledge, communication, clinical skills, and common sense. Clinical judgement requires practical experience, including observation of your own treatment failures, which may also be related in some instances to insufficient patient cooperation and motivation.

\section{STEP 1: COLLECT AND COLLATE INFORMATION}

\section{How can I help you?}

This initial phase in the treatment planning process begins by you asking the patient the simple but vital question: 'how can I help you?' This question is much more than a request for information, it is also an implication that from the outset you and your dental team are guided by values that set out, first and foremost, to help the patient. Trust and confidence are vital components of the dentist-patient relationship. Successful clinicians address the fundamental issue of trust by placing great emphasis on genuinely caring for patients in the widest sense of the word, by treating patients with respect, and by going to great lengths to communicate with them. A lack of adequate communication and trust has resulted in many unnecessary and unpleasant patient-dentist disagreements on treatments and their costs, and even in formal patient complaints to dental practice boards and legal proceedings.

\section{The patient's chief complaint(s) or problem(s)}

It is important to allow the patient time to explain, in his or her own words, the problem(s) as he or she sees it, as well as any expectations about possible treatment options and treatment outcomes. Resist the temptation to interrupt. Let the patient talk. Studies have shown that medical personnel allow patients very little time to 
express themselves, and also cannot resist the temptation to interrupt.

It has been said that treatment cannot be considered truly successful unless the patient is satisfied. You will experience situations where you feel that you have done a really excellent job, but where, for whatever reason, you have failed to match the patient's expectations. Many patients do not really have expectations of the technical care we provide (for example, how many patients would know if the distal margin of a full gold crown had an excellent adaptation?), but do hold expectations regarding pain control (did the dentist hurt me?), appearance (does this filling look okay?) and function (can I chew comfortably and effectively after treatment? Has the filling or crown stayed in place?).

However, a small number of patients hold what we might consider to be unrealistic expectations. Although we may wish to fulfil all of our patient's expectations, trying to do so for the patient with unrealistic demands usually courts disaster, as common sense and fundamental principles may often end up being ignored. If you honestly feel that the treatment you propose will not satisfy the patient, then it is preferable to say so from the outset. It is better to lose a new patient than to end up being involved later in possible litigation.

\section{History and examination}

The main purpose of the history and examination is to identify problems that may exist (such as dental, medical, psychological, social and financial problems), and to establish their relative importance to the patient and their effective management during subsequent treatments. History taking and clinical examination are two of the most important aspects of the patient assessment process, and complement each other to such an extent that it is impossible to build a satisfactory treatment plan without combining and collating information from the two procedures. For example, a diagnosis might appear self-evident by inspection alone. However, without an adequate case history the following may easily be overlooked:

- Coexistent diseases may go undetected and, therefore, untreated unless the patient is allowed to express his or her complaints
- A problem and its diagnosis, though seemingly obvious, may in reality be masking a serious and extensive disease which, again, will go untreated

- An assessment of the patient's attitudes and the establishment of good patient-clinician relationships are disregarded.

If previous records are available and you have not seen the patient before, then it is your professional responsibility to examine these records very carefully to note any previous problems or patient concerns, and the need for any follow-up such as medical conditions and treatments, pulp tests, radiographs and referrals. Have the original dental problems been satisfactorily treated? Update the medical and other histories - remember the adage 'never treat a stranger' (Sir William Ostler).

Collating the information gathered from the case history with that from the examination will enable the clinician to:

- Arrive at a tentative diagnosis of the patient's chief complaint(s) or problem(s)

- Determine any systemic factors that may influence this diagnosis

- Determine any systemic conditions that may require special precautions before and during dental procedures

- Compile a written record that will serve as legal evidence of professional competence.

\section{STEP 2: ESTABLISH DIAGNOSIS}

\section{Initial diagnosis}

An initial diagnosis of a problem can often be formed on the basis of the information gathered during the information collection phase, but it would be unusual to come to a definitive diagnosis without the use of one or more special tests. For example, a grossly carious tooth may seem the obvious cause of a patient's pain, but without taking radiographs it would be difficult to rule out the presence of other carious lesions, which also could be the source of the pain.

\section{Special investigations or tests}

These investigations provide information that cannot be gathered by means of:

- The history (thus, taking a medical history is not a special investigation, while taking a blood sample for

assessment is), and

- The examination using visual assessment, palpation and a probe (probing to measure pocket depth is not a special investigation, taking a periapical radiograph is).

Such tests have two functions. The first is as a screening tool to pick up unanticipated conditions, the second is as a means of confirming a provisional diagnosis. The panoramic radiograph is a good example of both functions. It can be used as a way of screening the patient for unerupted teeth, retained root fragments, unexpected bone cysts, tumours, etc., and as a way of confurming the position and morphology of, for example, a partially erupted third molar. Similarly, blood tests can be used to confirm the diagnosis of a particular condition such as anaemia, as well as a general screening tool. Dental diagnostic casts can be mounted in a semi-adjustable articulator and used to assess modifications to the patient's occlusion.

\section{Final or definitive diagnosis}

The final diagnosis of a problem(s), however simple or complex, only comes about once the history, clinical examination and various relevant special investigations have been conducted and the findings collated. The next stage is to consider the various options available to treat the patient and to choose the most appropriate after careful explanation.

\section{STEP 3: CONSIDER TREATMENT OPTIONS}

\section{List and assess the various options}

Enumerating the various alternative treatment options involves consideration of the mouth as a whole, not just individual teeth and, therefore, must also embrace structures such as the periodontium, soft tissues, and temporomandibular joints.

Practitioners should be conversant with the concept of evidence-based dentistry (EBD), which requires the judicious integration of systemic reviews of clinically relevant scientific evidence with the practitioner's clinical judgement and the dental treatment needs and preferences of the patient.

The following steps are required in the EBD process: 
- Define a clinically relevant and focused question

- Search for systematic reviews that may answer the question asked, such as the Cochrane Oral Health Group Reviews (www.ohg.cochrane.org/reviews)

- Evaluate the relevance of the evidence to the individual patient's needs.

All possible treatment options should be presented to the patient, who should be involved in deciding which of these options will ultimately be chosen, and their priorities. Some dentists favour presenting a single 'ideal' option, the one they see as providing the best possible result in terms of aesthetics and function, and then drawing attention to particular weaknesses of other possible options as compared to their favoured, supposedly ideal, course of action.

The problem with this normative need approach is that it fails to take into account the patient's dental healthcare wishes, beliefs and attitudes or behaviours, and it also fails to recognise that all treatment options possess both advantages and disadvantages. It places the dentist in the position of being the only person who knows what is 'good for' the patient, and may result in significant unnecessary treatment. Unfortunately also, the literature is replete with studies that have highlighted marked differences between practitoners in their diagnoses and preferred treatment plans for the same patient. What is relevant or significant to one practitioner may not be to another practitioner. Of course, the patient is more than likely to ask 'which option do you think is best?'. Your recommendation will draw on the following information gathered during the history and examination process.

\section{General considerations}

\section{The patient-dentist relationship}

Without due care and attention the likelihood of the dentist and the patient envisaging the same treatment expectations may not be high. There may be a dichotomy between what the patient wants and what the patient actually needs. Patients may be confused by dental terminology or jargon and fail to understand what is being proposed and why it is being proposed. They may have strong, but incorrect, assumptions about the reasons for their dental problems. Discussions in the area of relationships often focus on those situations characterised by a lack of rapport between the dentist and the patient, and clashes of personalities. It is clear that such a lack of harmony can and often does lead to ill-considered treatment plans. Thus, a dentist may find treating a particular patient so unpleasant that he or she recommends the least complex form of treatment, for example, extraction as opposed to root canal treatment and a crown. At the other extreme, clinicians should be wary of treating friends and family since they may end up proposing treatment plans that are too complex, for example, by advocating heroic measures to save a tooth which, in any other patient, they would consider unrestorable.

\section{The patient's beliefs about, and attitudes towards, dental care}

One course of treatment that may make complete sense for one patient may be unsuitable in another. For example, a 25-year-old female patient presents with pain from a previously restored mandibular first permanent molar. The diagnosis is irreversible pulpitis and the patient is very keen to retain the tooth, so the decision is made to treat the tooth endodontically and place a ceramometal crown. A second female patient of a similar age and an identical clinical condition is not bothered if she loses the tooth and so requests extraction. Two almost identical patients, but two very different treatment plans.

Often, a patient's negative beliefs and attitudes have been conditioned by previous unfortunate dental experiences. The patient may be very apprehensive following previous unpleasant or painful personal dental treatments, or by seeing such treatments experienced by others. In most instances, a caring attitude, a full explanation of the proposed dental procedure, and careful pain control techniques by the dentist are usually all that is required to encourage the patient to accept appropriate treatment. The patient should be placed semisupine in the dental chair (when this is possible) and reminded to relax tense shoulder and arm muscles while the warm local anaesthetic solution is being slowly administered. A simple procedure should first be chosen to gain the trust and confidence of the patient. Fearful patients are much less likely to return for routine dental treatments. Fortunately, relatively few patients require further sedation and/ or analgesic support methods once the 'fear of needles' and the fear of anticipated painful dental treatment procedures are overcome. Both hypnosis and relative analgesia using nitrous oxide sedation have been shown to be successful support methods in dentistry. Very apprehensive patients can benefit by writing down their earlier unpleasant dental experiences to share with the dentist, and then for them to analyse the reality of what is presently happening with what they feared may happen.

\section{The patient's age}

Clearly, the patient's age will have a bearing on many treatment decisions. In young patients the likelihood of further tooth eruption and large pulp chambers can impact on the decision whether or not to use a particular type of restoration to restore a tooth. In older patients the greater risks of periodontal disease and root caries can also play an important role in shaping the treatment plan. In either case, any advice given must be presented in a way that is appropriate to the patient's age.

\section{The patient's ability to tolerate dental treatment and to maintain any treatment provided}

A perfectly logical treatment plan may falter as a result of the patient's inability to tolerate treatment, especially where long treatment sessions are involved. The patient may simply not be able to open his or her mouth wide enough for you to gain sufficient access. Similarly, the patient may present with a neuromuscular condition that makes fine tooth preparation impossible. Such conditions also make the maintenance of oral hygiene that much more problematic, and it is unreasonable to expect all patients to be capable of effectively cleaning complex bridgework (fixed partial dentures).

\section{The patient's willingness to attend and their past attendance record}

Decisions on whether to leave alone, stabilise (and keep under review in either case) or to restore a tooth are often influenced by the degree of certainty as to whether the patient 
will attend in the future. The dentist who is confident that a particular patient will comply with requests to attend at certain time intervals will be more likely to defer restoring a tooth, prefering to keep it under review and ascertain the response to preventive measures rather than immediately place a permanent restoration. Work and family pressures, ill health, unemployment, and having to commute a long distance may cause problems in attending for treatment.

\section{The patient's financial considerations}

The cost of dental treatment cannot be left out of the treatment planning process, especially when the patient is expected to pay for all, or even part, of the treatment. Studies have shown that the cost of dental treatment may be less of a factor for patients than when and how the subject, and the making of payments, are discussed. However, costs become more significant with more complex treatment alternatives such as endodontic therapy versus tooth extraction, all-ceramic crown versus resin composite build-up, and acrylic resin removable partial denture versus ceramo-metal fixed partial denture or dental implant. Costs also may be incurred from taking time off from work. In private dental practice, costs are often closely linked to patient preferences and affordability, which then often determine the final treatment plan selected from several options. Affordability may also relate to the type of dental health insurance benefits or other third party reimbursement schemes that a patient may have for particular dental treatment costs.

Many dentists list the prices for individual items of treatment. Other dentists believe that the prices for individual items of treatment should not be shown to patients even if they are the basis upon which the fees have been set. Instead, the patient is quoted a global sum with the actual price list used merely as a reference by the dentist. Whichever approach is adopted, one common fundamental principle is that patients must be fully informed in writing of the probable costs of expensive procedures in particular before treatment is started.

\section{Your ability to perform a particular treatment}

A lack of experience in, for example, placing dental implants does not preclude the patient from this type of treatment, but will necessitate referring the patient to someone who does possess the required skills. Equally, you might be able to perform the treatment, but lack the necessary equipment and facilities to do so. Extend the services that you can offer by attending practical continuing education courses for proper training, and by investing in newer equipment and technology.

\section{Specific dental considerations}

These relate to the specific dental treatment being contemplated and include such factors as:

- Oral hygiene status, and motivation or cooperation of the patient

- Control of dental caries, tooth wear, and periodontal disease

- Pulpal and endodontic status of individual teeth

- Functional occlusal relationships and occlusal forces or stresses

- Appearance of the dentition

- Restoration retention (crown height, enamel available for bonding, etc).

\section{Risk assessment}

This stage of the treatment planning process is important and often done almost subconsciously. It requires the dentist to consider the likelihood of success or failure of the various treatment options as shown preferably by evidence-based studies, and to ensure that the patient fully understands the probable long-term prognosis for each option. Unfortunately, only limited information is available on the cost-effectiveness of restorative treatment options, which takes into account the long-term survival estimates of different treatments that have been discounted for their initial treatment costs. In addition, the survival of such treatments is influenced by the current oral disease risks present, such as those for dental caries, tooth surface loss and periodontal disease.

\section{STEP 4: FORMULATE TREATMENT PLAN}

\section{Select the most appropriate treatment option}

The aim of treatment is to provide a masticatory system that is functionally adequate, free from active disease and discomfort, and which is aesthetically pleasing to the patient. The objective of treatment planning is to identify problems of varying importance and urgency, to arrive at possible diagnoses, and to provide appropriate feasible solutions.

The most appropriate treatment option is the one which allows you to meet your professional ethical commitments, while at the same time satisfying the patient's needs and demands. In the majority of instances this happy balance will be easily achievable. As was described earlier though, problems arise when these two requirements are competing and cannot be reconciled. When in doubt, do not go ahead with providing treatment that you feel uncomfortable with, however much pressure you are subjected to by the patient.

\section{Formulate the treatment plan}

A well thought-out and documented treatment plan, tailored to the patient's needs and expectations, provides the following advantages:

- A smooth and logical progression through the various treatment phases

- Reduced stress for the patient, dentist and staff

- A more effective use of clinical time

- Less inconvenience for the patient

- A greater likelihood of achieving the patient's expectations

- The provision of essential information for the patient such as the time involved, costs, expected treatment outcomes and anticipated prognosis

- A reduced risk of non-payment of fees and of dento-legal problems

- The anticipation of any complications and the provision of contingency plans

- The ability of a colleague to continue, without difficulty, the treatment in your stead if required.

By the time the practitioner is able to present several possible treatment options having different advantages or benefits for the patient, a certain degree of trust and rapport should have been established. Communication should avoid the use of technical terms and overwhelming the patient with minute details. The dentist should first spend time in educating the patient about the causes, and the future prevention if relevant, of the dental problems diagnosed. Photographs, drawings, radiographs and diagnostic casts can assist in this education and explanation. 


\begin{tabular}{|c|c|}
\hline Treatment Phase & Treatment Items \\
\hline Emergency, and stabilisation & $\begin{array}{l}\text { Control of acute pain, infection, tooth fracture, gingival bleeding, } \\
\text { etc. Medical consultations if required. Extraction of non-strategic } \\
\text { hopeless teeth and retained roots, and caries and erosion control }\end{array}$ \\
\hline Preventive, and disease control & $\begin{array}{l}\text { Oral hygiene instruction }(\mathrm{OHI}) \text {, scaling and prophylaxis, fluorides, } \\
\text { chlorhexidine mouthrinses, casein-derived pastes, dietary analysis, } \\
\text { splint construction }\end{array}$ \\
\hline Initial restorative, and consultations & $\begin{array}{l}\text { Simple restorative work, placement of cores (foundations). } \\
\text { Endodontic, periodontic, prosthodontic, orthodontic, etc, } \\
\text { consultations }\end{array}$ \\
\hline Review, and occlusal analysis & $\begin{array}{l}\text { Assess responses to previous preventive and operative treatments. } \\
\text { Evaluate mounted casts and diagnostic wax-ups }\end{array}$ \\
\hline Definitive complex restorative & $\begin{array}{l}\text { Conformative or re-organised occlusal scheme? Need to increase the } \\
\text { occlusal vertical dimension and the clinical crown lengths? }\end{array}$ \\
\hline Maintenance, and monitoring & $\begin{array}{l}\text { Recalls: review oral hygiene and home care, periodontal health, } \\
\text { caries activity, occlusion, fixed and removable prostheses, implants. } \\
\text { Further radiographs and tests as required }\end{array}$ \\
\hline
\end{tabular}

Emphasise the benefits of the treatment proposed, and how it will help the patient. Confirm that the patient understands what you are saying, and is aware of the consequences of not having treatment or failing to take your advice regarding the prevention of similar future problems. The patient needs to be convinced that appropriate preventive and restorative treatments will resolve his or her dental problems with a satisfactory long-term successful and beneficial result. Possible barriers to treatment such as costs, time, and pain and discomfort must be discussed. Importantly, fully informed consent must be obtained from the patient or guardian before any treatment starts. Where treatment is likely to be expensive and provided over an extended period, then obtain the patient's signed agreement to the proposed plan and for stipulated periodic payments to be made.

Assuming that you feel confident that the end result of your treatment plan will satisfy the patient, the question remains, 'how to assign priority to, and to link in a logical manner, its various components?' The majority of patients accept the clinical advice of their dentist without question and, in most instances, the preventive, disease control and restorative treatment plan phases will be straightforward. Initially, a preventive and a disease control phase will usually be required in most new patients and to be reinforced in existing patients. The extent of these phases will vary considerably, and may be combined with any emergency and initial restorative treatments also required.

Often, restorative and initial subgingival scaling and root planing treatments are performed under local analgesia in the same quadrant at the same appointment. In most straightforward instances, whether a particular simple restoration is done first or last is usually of little consequence. However, the patient must be involved in the general planning process, partly as a courtesy and partly so that the patient feels he or she has a say in how things will be done, giving a sense of 'ownership' of his or her dental care.

Table 1 presents an overview of the treatment management process when complex restorative work is necessary, and acts as a guide for linking different components of the required treatment which, for convenience, may extend over a long period in some instances. No treatment plan is ever 'set in concrete', and treatment plans usually evolve over time to suit changing and sometimes unexpected situations. Trauma involving the teeth, and sudden deteriorations in general health, will have significant effects on proposed so-called 'final' treatment plans.

\section{OTHER SITUATIONS}

Not all patients will require an extensive plan. Some patients who seek urgent treatment will only be spending a brief time in your locality, and will not want any assessment and a comprehensive treatment treatment apart from usually the relief of discomfort or pain. These patients often have their own dentists from whom they will seek follow-up treatment. A medical history should be obtained to ensure that there are no systemic conditions present that would cause any problems from your dental treatment. Where appropriate, a brief note addressed to the patient's dentist and outlining what has been done may be given to the patient.

Regular patients will only require the updating of their medical and dental histories when they attend for recall. It is usually sufficient to enquire whether their medical status and any medications have changed since the last visit, and whether they have any dental problems that require attention. Patients can also check their previous documented medical and dental history questionnaire responses.

\section{READING}

Brennan D S, Spencer A J. Longitudinal comparison of factors influencing choice of dental treatment by private general practitioners. Aust Dent J 2006; 51: 117-123. Hackshaw A K, Paul E A, Davenport E S. EvidenceBased Dentistry. An Introduction. Oxford: Blackwell Munksgaard, 2006.

Jacobs D J, Steele J G, Wassell R M. Crowns and extracoronal restorations: Considerations when treatment planning. Br Dent J 2002; 192: 257-267.

Kay E J. Risks and values in treatment decision making. Prim Dent Care 2001; 8: 105-109.

Kay E J, Nuttall N M. Clinical Decision Making. An Art or a Science? London: British Dental Association, 1997. Kelly P G, Smales R J. Long-term costeffectiveness of single indirect restorations in selected dental practices. Br Dent J 2004; 196: 639-643.

Kronström M, Palmqvist S, Söderfeldt B. Prosthodontic decision making among general dentists in Sweden. I: The choice between crown therapy and filling. Int J Prosthodont 1999; 12: 426-431.

Kronström M, Palmqvist S, Söderfeldt B. Prosthodontic decision making among general dentists in Sweden. II: The choice between fixed and removable partial dentures. Int J Prosthodont 1999; 12: 527-533.

Kronström M, Palmqvist S, Söderfeldt B. Prosthodontic decision making among general dentists in Sweden. III: The choice between fixed partial dentures and single implants. Int J Prosthodont 2000; 13: 34-40.

Newsome P R H. The Patient Centred Dental Practice. London: British Dental Association, 2004.

Ryu J-I, Tsakos G, Sheiham A. Differences in prosthodontics treatment needs assessments between the standard normative and sociodental approach. Int J Prosthodont 2008; 21: 425-432.

Sanger R G, Boone M E. Problem-oriented dental record system - an alternative. In: Wood N K (ed) Treatment Planning. A Pragmatic Approach. St Louis: Mosby, 1978. p 323.

Smales R J. Practicing dentistry at different levels. Guest Editorial. J Pak Dent Assoc 2003; 12: 211-212.

Verdonschot E H, Plasschaert J M. Decision making in Restorative Dentistry. In Wilson NHF, Roulet J-F, Fuzzi M (eds) Advances in Operative Dentistry. Vol. 2: Challenges of the Future. Chicago: Quintessence, 2001. pp 75-87. 